Algebraic 83 Geometric $\mathcal{T}$ opology

Volume 4 (2004) 273-296

Published: 25 April 2004

ATG

\title{
A lower bound to the action dimension of a group
}

\author{
Sung YIL YOON
}

\begin{abstract}
The action dimension of a discrete group $\Gamma$, $\operatorname{actdim}(\Gamma)$, is defined to be the smallest integer $m$ such that $\Gamma$ admits a properly discontinuous action on a contractible $m$-manifold. If no such $m$ exists, we define $\operatorname{actdim}(\Gamma) \equiv \infty$. Bestvina, Kapovich, and Kleiner used Van Kampen's theory of embedding obstruction to provide a lower bound to the action dimension of a group. In this article, another lower bound to the action dimension of a group is obtained by extending their work, and the action dimensions of the fundamental groups of certain manifolds are found by computing this new lower bound.
\end{abstract}

AMS Classification 20F65; 57M60

Keywords Fundamental group, contractible manifold, action dimension, embedding obstruction

\section{Introduction}

Van Kampen constructed an $m$-complex that cannot be embedded into $\mathbb{R}^{2 m}[\underline{8}$. A more modern approach to Van Kampen's theory of embedding obstruction uses co/homology theory. To see the main idea of this co/homology theoretic approach, let $K$ be a simplicial complex and $|K|$ denote its geometric realization. Define the deleted product

$$
|\tilde{K}| \equiv\{(x, y) \in|K| \times|K| \mid x \neq y\}
$$

such that $\mathbb{Z}_{2}$ acts on $|\tilde{K}|$ by exchanging factors. Observe that there exists a two-fold covering $|\tilde{K}| \rightarrow|\tilde{K}| / \mathbb{Z}_{2}$ with the following classifying map:

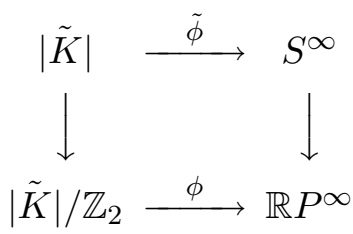


Now let $\omega^{m} \in H^{m}\left(\mathbb{R} P^{\infty} ; \mathbb{Z}_{2}\right)$ be the nonzero class. If $\phi^{*}\left(w^{m}\right) \neq 0$ then $|K|$ cannot be embedded into $\mathbb{R}^{m}$. That is, there is $\Sigma^{m} \in H_{m}\left(|K| / \mathbb{Z}_{2} ; \mathbb{Z}_{2}\right)$ such that $\left\langle\phi^{*}\left(w^{m}\right), \Sigma\right\rangle \neq 0$.

A similar idea was used to obtain a lower bound to the action dimension of a discrete group $\Gamma$ 2]. Specifically, the obstructor dimension of a discrete group $\Gamma, \operatorname{obdim}(\Gamma)$, was defined by considering an $m$-obstructor $K$ and a proper, Lipschitz, expanding map

$$
f: \operatorname{cone}(K)^{(0)} \rightarrow \Gamma
$$

And it was shown that

$$
\operatorname{obdim}(\Gamma) \leq \operatorname{actdim}(\Gamma) .
$$

See 2] for details. An advantage of considering $\operatorname{obdim}(\Gamma)$ becomes clear when $\Gamma$ has well-defined boundary $\partial \Gamma$, for example, when $\Gamma$ is $C A T(0)$ or torsion free hyperbolic. In these cases, if an $m$-obstructor $K$ is contained in $\partial \Gamma$ then $m+2 \leq \operatorname{obdim}(\Gamma)$.

If $\Gamma$ acts on a contractible $m$-manifold $W$ properly discontinuously and cocompactly, then it is easy to see that $\operatorname{actdim}(\Gamma)=m$. For example, let $M$ be a Davis manifold. That is, $M$ is a closed, aspherical, four-dimensional manifold whose universal cover $\tilde{M}$ is not homeomorphic to $\mathbb{R}^{4}$. We know that $\operatorname{actdim}\left(\pi_{1}(M)\right)=4$. However, it is not easy to see that $\operatorname{obdim}\left(\pi_{1}(M)\right)=4$. The goal of this article is to generalize the definitions of obstructor and obstructor dimension. To do so, we define proper obstructor (Definition 2.5) and proper obstructor dimension (Definition [5.2.) The main result is the following.

Main Theorem The proper obstructor dimension of $\Gamma \leq \operatorname{actdim}(\Gamma)$.

As applications we will answer the following problems:

- Suppose $W$ is a closed aspherical manifold and $\tilde{W}$ is its universal cover so that $\pi_{1}(W)$ acts on $\tilde{W}$ properly discontinuously and cocompactly. We show that $\tilde{W}$ in this case is indeed an $m$-proper obstructor and $\operatorname{pobdim}\left(\pi_{1}(W)\right)=m$.

- Suppose $W_{i}$ is a compact aspherical $m_{i}$-manifold with all boundary components aspherical and incompressible, $i=1, \ldots, d$. (Recall that a boundary component $N$ of a manifold $W$ is called incompressible if $i_{*}: \pi_{j}(N) \rightarrow \pi_{j}(W)$ is injective for $j \geq 1$.) Also assume that for each $i, 1 \leq i \leq d$, there is a component of $\partial W_{i}$, call it $N_{i}$, so that $\left|\pi_{1}\left(W_{i}\right): \pi_{1}\left(N_{i}\right)\right|>2$. Let $G=\pi_{1}\left(W_{1}\right) \times \ldots \times \pi_{1}\left(W_{d}\right)$. Then

$$
\operatorname{actdim}(G)=m_{1}+\ldots+m_{d} \text {. }
$$


The organization of this article is as follows. In Section 2, we define proper obstructor. The coarse Alexander duality theorem by Kapovich and Kleiner [5], is used to construct the first main example of proper obstructor in Section [3, Several examples of proper obstructors are constructed in Section 4 . Finally, the main theorem is proved and the above problems are considered in Sections 5 .

\section{Proper obstructor}

To work in the PL-category we define simplicial deleted product

$$
\tilde{K} \equiv\{\sigma \times \tau \in K \times K \mid \sigma \cap \tau=\emptyset\}
$$

such that $\mathbb{Z}_{2}$ acts on $\tilde{K}$ by exchanging factors. It is known that $|\tilde{K}| / \mathbb{Z}_{2}(|\tilde{K}|)$ is a deformation retract of $\tilde{K} / \mathbb{Z}_{2}(\tilde{K})$, see [7, Lemma 2.1]. Therefore, WLOG, we can use $H_{m}\left(\tilde{K} / \mathbb{Z}_{2} ; \mathbb{Z}_{2}\right)$ instead of $H_{m}\left(|\tilde{K}| / \mathbb{Z}_{2} ; \mathbb{Z}_{2}\right)$.

Throughout the paper, all homology groups are taken with $\mathbb{Z}_{2}$-coefficients unless specified otherwise.

To define proper obstructor, we need to consider several definitions and preliminary facts.

Definition 2.1 A proper map $h: A \rightarrow B$ between proper metric spaces is uniformly proper if there is a proper function $\phi:[0, \infty) \rightarrow[0, \infty)$ such that

$$
d_{B}(h(x), h(y)) \geq \phi\left(d_{A}(x, y)\right)
$$

for all $x, y \in A$. (Recall that a metric space is said to be proper if any closed metric ball is compact, and a map is said to be proper if the preimages of compact sets are compact.)

Let $W$ be a contractible $m$-manifold and define

$$
W_{0} \equiv\{(x, y) \in W \times W \mid x \neq y\} .
$$

Consider a uniformly proper map $\beta: Y \rightarrow W$ where $Y$ is a simplicial complex and $W$ is a contractible manifold. Since $\beta$ is uniformly proper, we can choose $r>0$ such that $\beta(a) \neq \beta(b)$ if $d_{Y}(a, b)>r$. Note that $\beta$ induces an equivariant map:

$$
\bar{\beta}:\left\{\left(y, y^{\prime}\right) \in Y \times Y \mid d_{Y}\left(y, y^{\prime}\right)>r\right\} \rightarrow W_{0}
$$

As we work in the PL-category we make the following definition. 
Definition 2.2 If $K \subset Y$ is a subcomplex and $r$ is a positive integer then we define the combinatorial $r$-tubular neighborhood of $K$, denoted by $N_{r}(K)$, to be $r$-fold iterated closed star neighborhood of $K$.

Recall that when $Y$ is a simplicial complex, $|Y| \times|Y|$ can be triangulated so that each cell $\sigma \times \tau$ is a subcomplex. Let $d: Y \rightarrow Y^{2}$ be the diagonal map, $d(\sigma)=(\sigma, \sigma)$, where $Y^{2}$ is triangulated so that $d(Y)$ is a subcomplex. Define

$$
Y_{r} \equiv C l s\left(Y^{2}-N_{r}(d(Y))\right) \text {. }
$$

Note that a uniformly proper map $\beta: Y \rightarrow W^{m}$ induces an equivariant map $\bar{\beta}: Y_{r} \rightarrow W_{0} \simeq S^{m-1}$ for some $r>0$.

Definition 2.3 (Essential $\mathbb{Z}_{2}-m$-cycle) An essential $\mathbb{Z}_{2}-m$-cycle is a pair $\left(\tilde{\Sigma}^{m}, a\right)$ satisfying the following conditions:

(i) $\quad \tilde{\Sigma}^{m}$ is a finite simplicial complex such that $\left|\tilde{\Sigma}^{m}\right|$ is a union of $m$-simplices and every $(m-1)$-simplex is the face of an even number of $m$-simplices.

(ii) $a: \tilde{\Sigma}^{m} \rightarrow \tilde{\Sigma}^{m}$ is a free involution.

(iii) There is an equivariant map $\varphi: \tilde{\Sigma}^{m} \rightarrow S^{m}$ with $\operatorname{deg}(\varphi)=1(\bmod 2)$.

Some remarks are in order.

(1) We recall how to find $\operatorname{deg}(\varphi)$. Choose a simplex $s$ of $S^{m}$ and let $f$ be a simplicial approximation to $\varphi$. Then $\operatorname{deg}(\varphi)$ is the number of $m-$ simplices of $\tilde{\Sigma}^{m}$ mapped into $s$ by $f$.

(2) Let $\tilde{\sigma}$ be the sum of all $m$-simplices of $\tilde{\Sigma}^{m}$. Condition (i) of Definition 2.3 implies that $[\tilde{\sigma}] \in H_{m}\left(\tilde{\Sigma}^{m}\right)$. We call $[\tilde{\sigma}]$ the fundamental class of $\tilde{\Sigma}^{m}$.

(3) Let $\tilde{\Sigma}^{m} / \mathbb{Z}_{2} \equiv \Sigma^{m}$ and consider a two-fold covering $q: \tilde{\Sigma}^{m} \rightarrow \Sigma^{m}$. As $\varphi$ is equivariant it induces $\bar{\varphi}: \Sigma^{m} \rightarrow \mathbb{R} P^{m}$. Let $\operatorname{deg}_{2}(\varphi)$ denote $\operatorname{deg}(\varphi)(\bmod 2)$.

Note that $\operatorname{deg}_{2}(\varphi)=\left\langle\bar{\varphi}^{*}\left(w^{m}\right), q \tilde{\sigma}\right\rangle$ where $w^{m} \in H^{m}\left(\mathbb{R} P^{m} ; \mathbb{Z}_{2}\right)$ is the nonzero element. If $\varphi: \tilde{\Sigma}^{m} \rightarrow S^{m}$ is an equivariant map then $\operatorname{deg}_{2}(\varphi)=$ 1. To see this, we prove the following proposition.

Proposition 2.4 Suppose a map $\varphi: \tilde{\Sigma}^{m} \rightarrow S^{m}$ is equivariant. Then

$$
\operatorname{deg}_{2}(\varphi)=1
$$


Proof Consider the classifying map and the commutative diagram for a twofold covering $q: \tilde{\Sigma}^{m} \rightarrow \Sigma^{m}$ :

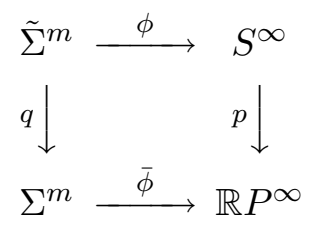

We also have:

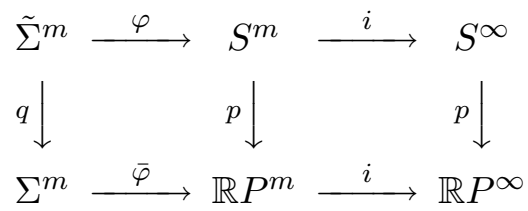

Because $S^{\infty} \rightarrow \mathbb{R} P^{\infty}$ is the classifying covering, $i \circ \varphi \simeq \phi$ and $i \circ \bar{\varphi} \simeq \bar{\phi}$.

Observe that

$$
\operatorname{deg}_{2}(\varphi)=\left\langle\bar{\varphi}^{*} w^{m}, q \tilde{\sigma}\right\rangle=\left\langle(i \circ \bar{\varphi})^{*} w_{\infty}^{m}, q \tilde{\sigma}\right\rangle
$$

where $0 \neq w_{\infty}^{m} \in H^{m}\left(\mathbb{R} P^{\infty}\right)$. But, since $i \circ \bar{\varphi} \simeq \bar{\phi}$,

$$
\left\langle(i \circ \bar{\varphi})^{*} w_{\infty}^{m}, q \tilde{\sigma}\right\rangle=\left\langle\bar{\phi}^{*} w_{\infty}^{m}, q \tilde{\sigma}\right\rangle=\operatorname{deg}_{2}(\phi) .
$$

Now we modify the definition of obstructor.

Definition 2.5 (Proper obstructor) Let $T$ be a contractible 1 simplicial complex. Recall that $T_{r} \equiv C l s\left(T^{2}-N_{r}(d(T))\right)$ where $N_{r}(d(T))$ denotes the $r$ tubular neighborhood of the image of the diagonal map $d: T \rightarrow T^{2}$. Let $m$ be the largest integer such that for any $r>0$, there exists an essential $\mathbb{Z}_{2}-m-$ cycle $\left(\tilde{\Sigma}^{m}, a\right)$ and a $\mathbb{Z}_{2}$-equivariant map $f: \tilde{\Sigma}^{m} \rightarrow T_{r}$. If such $m$ exists then $T$ is called an $m$-proper obstructor.

The first example of proper obstructor is given by the following proposition.

Proposition 2.6 Suppose that $M$ is a $k$-dimensional closed aspherical manifold where $k>1$ and $X$ is the universal cover of $M$. Suppose also that $X$ has a triangulation so that $X$ is a metric simplicial complex and a group $G=\pi_{1}(M)$ acts on $X$ properly discontinuously, cocompactly, simplicially, and freely by isometries. Then $X^{k}$ is a $(k-1)$-proper obstructor.

We prove Proposition 2.6 in Section 3. The key ideas are the following:

\footnotetext{
${ }^{1}$ Contractibility is necessary for Proposition 5.2
} 
(1) Since $G$ acts on $X$ properly discontinuously, cocompactly, simplicially, and freely by isometries, $X$ is uniformly contractible. Recall that a metric space $Y$ is uniformly contractible if for any $r>0$, there exists $R>r$ such that $B_{r}(y)$ is contractible in $B_{R}(y)$ for any $y \in Y$.

(2) For any $R>0$, there exists $R^{\prime}>R$ so that the inclusion induced map

$$
i_{*}: \tilde{H}_{j}\left(X_{R^{\prime}}\right) \rightarrow \tilde{H}_{j}\left(X_{R}\right)
$$

is trivial for $j \neq k-1$ and $\mathbb{Z}_{2} \cong i_{*}\left(\tilde{H}_{k-1}\left(X_{R^{\prime}}\right)\right) \leq \tilde{H}_{k-1}\left(X_{R}\right)$. (See Lemma 3.6])

(3) We recall the definition of $\Delta$-complex and use it to complete the proof as sketched below.

Definition 2.7 A $\Delta$-complex is a quotient space of a collection of disjoint simplices of various dimensions, obtained by identifying some of their faces by the canonical linear homeomorphisms that preserve the ordering of vertices.

Suppose $\left(\tilde{\Sigma}^{m}, a\right)$ is an essential $\mathbb{Z}_{2}-m$-cycle with a $\mathbb{Z}_{2}$-equivariant map $f:\left(\tilde{\Sigma}^{m}, a\right) \rightarrow T_{r}$. Let

$$
\left|\tilde{\Sigma}^{m}\right|=\cup_{i=1}^{n} \Delta_{i}^{m}
$$

(union of $n$-copies of $m$-simplices, use subscripts to denote different copies of $m$-simplices) and

$$
\left.f_{i} \equiv f\right|_{\Delta_{i}^{m}}
$$

Then condition (i) of Definition 2.3 implies that $\sum_{i=1}^{n} f_{i}$ is an $m$-cycle of $T_{r}$ (over $\mathbb{Z}_{2}$ ). That is, an essential $\mathbb{Z}_{2}-m$-cycle $\left(\tilde{\Sigma}^{m}, a\right)$ with a $\mathbb{Z}_{2}$-equivariant map $f:\left(\tilde{\Sigma}^{m}, a\right) \rightarrow T_{r}$ can be considered as an $m$-cycle of $T_{r}$ (over $\mathbb{Z}_{2}$ ). Next suppose that $g=\sum_{i=1}^{n} g_{i}$ is an $m$-chain of $T_{r}$ (over $\mathbb{Z}_{2}$ ) where $g_{i}: \Delta^{m} \rightarrow T_{r}$ are singular $m$-simplices. Take an $m$-simplex for each $i$ and index them as $\Delta_{i}^{m}$. Let $\Delta_{i}^{m-1}$ denote a codimension 1 face of $\Delta_{i}^{m}$. Construct a $\Delta$-complex $\Pi$ as follows:

- $|\Pi|=\cup_{i=1}^{n} \Delta_{i}^{m}$

- For each $\ell \neq j$ we identify $\Delta_{\ell}^{m}$ with $\Delta_{j}^{m}$ along $\Delta_{\ell}^{m-1}$ and $\Delta_{j}^{m-1}$ whenever $\left.g_{l}\right|_{\Delta_{\ell}^{m-1}}=\left.g_{j}\right|_{\Delta_{j}^{m-1}}$.

Subdivide $\Pi$ if necessary so that $\Pi$ becomes a simplicial complex. Consider when $g$ is an $m$-cycle and an $m$-boundary.

First, suppose $g$ is an $m$-cycle. Then for any codimension 1 face $\Delta_{i}^{m-1}$ of $\Delta_{i}^{m}$ there are an even number of $j$ 's(including $i$ itself) between 1 and $n$ such 
that $\left.g\right|_{\Delta_{i}^{m-1}}=\left.g\right|_{\Delta_{j}^{m-1}}$ So $\Pi$ satisfies condition (i) of Definition 2.3 and we can consider $g$ as a map

$$
g: \Pi \rightarrow T_{r}
$$

by setting $\left.g\right|_{\Delta_{i}^{m}}=g_{i}$.

Second, suppose $g$ is an $m$-boundary. Then there is an $(m+1)$-chain $G \equiv$ $\sum_{i=1}^{N} G_{i}$ where $G_{i}: \Delta^{m+1} \rightarrow T_{r}$ are singular $(m+1)$-simplices such that $\partial G=$ $g$. As before one can construct a simplicial complex $\Omega$ and consider $G$ as a map

$$
G: \Omega \rightarrow T_{r}
$$

Let $\partial \Omega \equiv \cup\{m$ - simplices of $\Omega$ which are the faces of an odd number of $(m+1)$ simplices $\}$. Note that $\partial \Omega \stackrel{\text { comb. }}{\cong} \Pi$ where $\stackrel{c o m b}{\cong}$ denotes combinatorial equivalence. This observation will be used to construct an essential cycle in the proof of Proposition 2.6.

\section{Coarse Alexander duality}

We first review the terminology of [5]. Some terminology already defined is modified in the PL category. Let $X$ be (the geometric realization of) a locally finite simplicial complex. We equip the 1 -skeleton $X^{(1)}$ with path metric by defining each edge to have unit length. We call such an $X$ with the metric on $X^{(1)}$ a metric simplicial complex. We say that $X$ has bounded geometry if all links have a uniformly bounded number of simplices. Recall that $X_{r} \equiv$ $C l s\left(X^{2}-N_{r}(d(X))\right)$, see Definition 2.2. Also denote:

$$
\left\{\begin{array}{l}
B_{r}(c) \equiv\{x \in X \mid d(c, x) \leq r\} \\
\partial B_{r}(c) \equiv\{x \in X \mid d(c, x)=r\}
\end{array}\right.
$$

If $C_{*}(X)$ is the simplicial chain complex and $A \subset C_{*}(X)$ then the support of $A$, denoted by Support $(A)$, is the smallest subcomplex of $K \subset X$ such that $A \subset C_{*}(K)$. We say that a homomorphism

$$
h: C_{*}(X) \rightarrow C_{*}(X)
$$

is coarse Lipschitz if for each simplex $\sigma \subset X$, Support $\left(h\left(C_{*}(\sigma)\right)\right)$ has uniformly bounded diameter. We call a coarse Lipschitz map with

$$
D \equiv \max _{\sigma} \operatorname{diam}\left(\operatorname{Support}\left(h\left(C_{*}(\sigma)\right)\right)\right)
$$

$D$-Lipschitz. We call a homomorphism $h$ uniformly proper, if it is coarse Lipschitz and there exists a proper function $\phi: \mathbb{R}_{+} \rightarrow \mathbb{R}$ so that for each subcomplex $K \subset X$ of diameter $\geq r$, Support $\left(h\left(C_{*}(\sigma)\right)\right)$ has diameter $\geq \phi(r)$. 
We say that a homomorphism $h$ has displacement $\leq D$ if for every simplex $\sigma \subset X$, Support $\left(h\left(C_{*}(\sigma)\right)\right) \subset N_{D}(\sigma)$. A metric simplicial complex is uniformly acyclic if for every $R_{1}$ there is an $R_{2}$ such that for each subcomplex $K \subset X$ of diameter $\leq R_{1}$ the inclusion $K \rightarrow N_{R_{2}}(K)$ induces zero on reduced homology groups.

Definition 3.1 (PD group) A group $\Gamma$ is called an $n$-dimensional Poincaré duality group $(P D(n)$ group in short) if the following conditions are satisfied:

(i) $\Gamma$ is of type $F P$ and $n=\operatorname{dim}(\Gamma)$.

(ii) $H^{j}(\Gamma ; \mathbb{Z} \Gamma)= \begin{cases}0 & j \neq n \\ \mathbb{Z} & j=n\end{cases}$

Example 3.2 The fundamental group of a closed aspherical $k$-manifold is a $P D(k)$ group. See [3] for details.

Definition 3.3 (Coarse Poincaré duality space [5]) A Coarse Poincaré duality space of formal dimension $k, P D(k)$ space in short, is a bounded geometry metric simplicial complex $X$ so that $C_{*}(X)$ is uniformly acyclic, and there is a constant $D_{0}$ and chain mappings

$$
C_{*}(X) \stackrel{\bar{P}}{\rightarrow} C_{c}^{k-*}(X) \stackrel{P}{\rightarrow} C_{*}(X)
$$

so that

(i) $P$ and $\bar{P}$ have displacement $\leq D_{0}$,

(ii) $P \circ \bar{P}$ and $\bar{P} \circ P$ are chain homotopic to the identity by $D_{0}$-Lipschitz chain homotopies $\Phi: C_{*}(X) \rightarrow C_{*+1}(X), \bar{\Phi}: C_{c}^{*}(X) \rightarrow C_{c}^{*-1}(X)$. We call coarse Poincare duality spaces of formal dimension $\mathrm{k}$ a coarse $P D(k)$ spaces.

Example 3.4 An acyclic metric simplicial complex that admits a free, simplicial cocompact action by a $P D(k)$ group is a coarse $P D(k)$ space.

For the rest of the paper, let $X$ denote the universal cover of a $k$-dimensional closed aspherical manifold where $k>1$.

Assume also that $X$ has a triangulation so that $X$ is a metric simplicial complex with bounded geometry, and $G=\pi_{1}(M)$ acts on $X$ properly discontinuously, cocompactly, simplicially, and freely by isometries. In particular, $G$ is a $P D(k)$ group and $X$ is a coarse $P D(k)$ space. The following theorem was proved in [5]. Pro-Category theory is reviewed in Appendix A. 
Theorem 3.5 (Coarse Alexander duality [5]) Suppose $Y$ is a coarse $P D(n)$ space, $Y^{\prime}$ is a bounded geometry, uniformly acyclic metric simplicial complex, and $f: C_{*}\left(Y^{\prime}\right) \rightarrow C_{*}(Y)$ is a uniformly proper chain map. Let $K \equiv$ $\operatorname{Support}\left(f\left(C_{*}\left(Y^{\prime}\right)\right)\right), Y_{R} \equiv C l s\left(Y-N_{R}(K)\right)$. Then we can choose $0<r_{1}<$ $r_{2}<r_{3}<\ldots$ and define the inverse system $\operatorname{pro}_{j}\left(Y_{r}\right) \equiv\left\{\tilde{H}_{j}\left(Y_{r_{i}}\right), i_{*}, \mathbb{N}\right\}$ so that

$$
\operatorname{pro} \tilde{H}_{n-j-1}\left(Y_{r}\right) \cong H_{c}^{j}\left(Y^{\prime}\right) \text {. }
$$

We rephrase the coarse Alexander duality theorem.

Lemma 3.6 Recall that $X$ is a metric simplicial complex with bounded geometry and a group $G$ acts on $X$ properly discontinuously, cocompactly, simplicially, and freely by isometries. Also recall that $X_{r} \equiv C l s\left(X^{2}-N_{r}(d(X))\right)$. One can choose $0<r_{1}<r_{2}<r_{3}<\ldots$ and define the inverse system $\operatorname{pro}_{j}\left(X_{r}\right)$ $\equiv\left\{\tilde{H}_{j}\left(X_{r_{i}}\right), i_{*}, \mathbb{N}\right\}$ so that:

$$
\operatorname{pro}_{j}\left(X_{r}\right)= \begin{cases}\mathbf{0}, & j \neq k-1 \\ \mathbb{Z}_{2}, & j=k-1\end{cases}
$$

Proof Consider the diagonal map

$$
d: X \rightarrow X^{2}, x \mapsto(x, x)
$$

and note that $d$ is uniformly proper and $X^{2}$ is a $P D(2 k)$ space. Theorem 3.5 implies that

$$
\operatorname{pro} \tilde{H}_{2 k-*-1}\left(X_{r}\right)=H_{c}^{*}(X) .
$$

Finally observe that $H_{c}^{*}(X) \cong H_{k-*}\left(\mathbb{R}^{k}\right) \cong H_{c}^{*}\left(\mathbb{R}^{k}\right)$.

Now we prove Proposition 2.6 .

Proof of Proposition 2.6 Let $r>0$ be given. First use Lemma 3.6 to choose $r=r_{1}<r_{2}<\ldots<r_{k-1}<r_{k}$ so that

$$
i_{*}: \tilde{H}_{j}\left(X_{r_{m+1}}\right) \rightarrow \tilde{H}_{j}\left(X_{r_{m}}\right)
$$

is trivial for $j \neq k-1$. In particular, $i: X_{r_{k}} \rightarrow X_{r_{k-1}}$ is trivial in $\pi_{0}$. Let $S^{0} \equiv\{e, w\}$ and define an involution $a_{0}$ on $S^{0}$ by $a_{0}(e)=w$ and $a_{0}(w)=e$. Let $\theta:\left(S^{0}, a_{0}\right) \rightarrow\left(X_{r_{k}}, s\right)$ be an equivariant map where $s$ is the obvious involution on $X_{r_{i}}$. Now let

$$
\sigma: I \rightarrow X_{r_{k-1}}
$$

be a path so that $\sigma(0)=\theta(e)$ and $\sigma(1)=\theta(w)$. Define

$$
\sigma^{\prime}: I \rightarrow X_{r_{k-1}}
$$




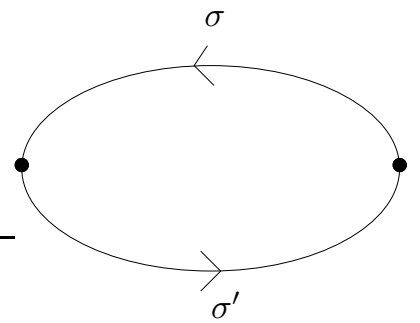

Figure 1: $\sigma_{1}$

by $\sigma^{\prime}(t)=s \sigma(t)$. Observe that $\sigma_{1} \equiv \sigma+\sigma^{\prime}$ is an $1-$ cycle in $X_{r_{k-1}}$. See Figure [1.

Let $a_{1}$ be the obvious involution on $S^{1}$ and consider $\sigma_{1}$ as an equivariant map

$$
\sigma_{1}:\left(S^{1}, a_{1}\right) \rightarrow\left(X_{r_{k-1}}, s\right) .
$$

Since $i_{*}: \tilde{H}_{1}\left(X_{r_{k-1}}\right) \rightarrow \tilde{H}_{1}\left(X_{r_{k-2}}\right)$ is trivial, $\sigma_{1}$ is the boundary of a 2 -chain in $X_{r_{k-2}}$. Call this 2 -chain $\sigma_{2}^{+}=\sum_{i=1}^{m} g_{i}$ where $g_{i}$ are singular 2-simplices. Following Remark (3) after Proposition [2.6] construct a simplicial complex $\tilde{\Sigma}_{+}^{2}$ such that

$$
\sigma_{2}^{+}: \tilde{\Sigma}_{+}^{2} \rightarrow X_{r_{k-2}} \text { and } \partial \sigma_{2}^{+}=\sigma_{1} .
$$

See Figure 2. Define the boundary of $\tilde{\Sigma}_{+}^{2}, \partial \tilde{\Sigma}_{+}^{2}$, to be the union of 1-simplices, which are the faces of an odd number of 2 -simplices. Recall also from Remark (3) that $\partial \tilde{\Sigma}_{+}^{2} \stackrel{\text { comb. }}{\cong} S^{1}$ where $\stackrel{\text { comb. }}{\cong}$ denotes combinatorial equivalence.

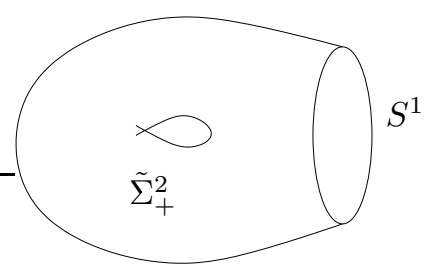

Figure 2: $\tilde{\Sigma}_{+}^{2}$

Next, let $\sigma_{2}^{-}=s \sigma_{2}^{+}=\sum_{i=1}^{m} s g_{i}$. Take a copy of $\tilde{\Sigma}_{+}^{2}$, denoted by $\tilde{\Sigma}_{-}^{2}$, such that

$$
\sigma_{2}^{-}: \tilde{\Sigma}_{-}^{2} \rightarrow X_{r_{k-2}} \text { and } \partial \sigma_{2}^{-}=\sigma_{1} \text {. }
$$

Construct $\tilde{\Sigma}^{2}$ by attaching $\tilde{\Sigma}_{+}^{2}$ and $\tilde{\Sigma}_{-}^{2}$ along $S^{1}=\partial \tilde{\Sigma}_{+}^{2}=\partial \tilde{\Sigma}_{-}^{2}$ by identifying $x \sim a_{1}(x)$. That is, $\tilde{\Sigma}^{2} \equiv \tilde{\Sigma}_{+}^{2} \cup_{S^{1}} \tilde{\Sigma}_{-}^{2}$. See Figure 3 Define an involution $a_{2}$ 


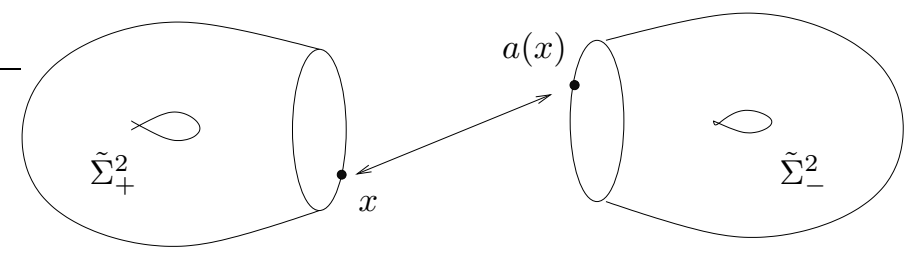

Figure 3: Constructing $\tilde{\Sigma}^{2}$

on $\tilde{\Sigma}^{2}$ by setting

$$
a_{2}(x)=\left\{\begin{array}{lll}
x \in \tilde{\Sigma}_{+}^{2} & \text { if } & x \in \tilde{\Sigma}_{-}^{2}-S^{1} \\
x \in \tilde{\Sigma}_{-}^{2} & \text { if } & x \in \tilde{\Sigma}_{+}^{2}-S^{1} \\
a_{1}(x) & \text { if } x \in S^{1}
\end{array}\right.
$$

Observe that $\sigma_{2} \equiv \sigma_{2}^{+}+\sigma_{2}^{-}$is a 2 -cycle in $X_{r_{2}}$ and we can consider $\sigma_{2}$ as an equivariant map

$$
\sigma_{2}:\left(\tilde{\Sigma}^{2}, a_{2}\right) \rightarrow\left(X_{r_{k-2}}, s\right) .
$$

Continue inductively and construct a $(k-1)$-cycle

$$
\sigma_{k-1}:\left(\tilde{\Sigma}^{k-1}, a_{k-1}\right) \rightarrow\left(X_{r_{1}}=X_{r_{k-(k-1)}}, s\right)^{\prime}
$$

Simply write $a$ instead of $a_{k-1}$, and note that $X_{r_{1}} \subset X_{r}$. So $\left(\tilde{\Sigma}^{k-1}, a\right)$ satisfies conditions (i)-(ii) of Definition 2.3 and we only need to show that it satisfies condition (iii).

It was proved in 2] that there exists a $\mathbb{Z}_{2}$-equivariant homotopy equivalence $\tilde{h}: X_{0} \rightarrow S^{k-1}$. So $\tilde{h}$ induces a homotopy equivalence

$$
h: X_{0} / \sim \rightarrow \mathbb{R} P^{k-1} .
$$

Let $g \equiv h i \sigma_{k-1}: \tilde{\Sigma}^{k-1} \stackrel{\sigma_{k-1}}{\rightarrow} X_{r} \stackrel{i}{\rightarrow} X_{0} \stackrel{h}{\rightarrow} S^{k-1}$. Note that $g$ is equivariant. We shall prove that $\operatorname{deg}(g)=1(\bmod 2)$ by constructing another map

$$
f_{k-1}: \tilde{\Sigma}^{k-1} \rightarrow S^{k-1}
$$

with odd degree and applying Proposition 2.4.

Observe that

$$
S^{1} \subset \tilde{\Sigma}^{2} \subset \tilde{\Sigma}^{3} \subset \ldots \subset \tilde{\Sigma}^{k-2} \subset \tilde{\Sigma}^{k-1}
$$

and for each $i, 2 \leq i \leq k-1$ :

$$
\tilde{\Sigma}^{i}=\tilde{\Sigma}_{+}^{i} \cup_{\tilde{\Sigma}^{i-1}} \tilde{\Sigma}_{-}^{i}
$$


Now construct a map $f_{k-1}: \tilde{\Sigma}^{k-1} \rightarrow S^{k-1}$ as follows: First let $f_{1}: S^{1} \rightarrow S^{1}$ be the identity and extend $f_{1}$ to $f_{2}^{+}: \tilde{\Sigma}_{+}^{2} \rightarrow B^{2}$ by Tietze Extension theorem. Without loss of generality assume that $\left(f_{2}^{+}\right)^{-1}\left(S^{1}\right) \subset S^{1} \stackrel{\text { comb. }}{\cong} \partial \tilde{\Sigma}_{+}^{2}$. Then extend equivariantly to $f_{2}: \tilde{\Sigma}^{2} \rightarrow S^{2}$. Note that $f_{2}^{-1}\left(B_{+}^{2}\right) \subset \tilde{\Sigma}_{+}^{2}, f_{2}^{-1}\left(B_{-}^{2}\right) \subset$ $\tilde{\Sigma}_{-}^{2}$, and $f_{2}^{-1}\left(S^{1}\right) \subset S^{1}$.

Continue inductively and construct an equivariant map

$$
f_{k-1}: \tilde{\Sigma}^{k-1} \rightarrow S^{k-1} .
$$

By construction, we know that

$$
f_{j}^{-1}\left(B_{+}^{j}\right) \subset \tilde{\Sigma}_{+}^{j}, f_{j}^{-1}\left(B_{-}^{j}\right) \subset \tilde{\Sigma}_{-}^{j}, \text { and } f_{j}^{-1}\left(S^{j-1}\right) \subset \tilde{\Sigma}^{j-1}, 2 \leq j \leq k-1 .
$$

Observe that $\operatorname{deg}\left(f_{k-1}\right)=\operatorname{deg}\left(f_{k-2}\right)=\ldots=\operatorname{deg}\left(f_{2}\right)=\operatorname{deg}\left(f_{1}\right)$. (Recall that $\operatorname{deg}\left(f_{m}\right) \equiv$ the number of $m$-simplices of $\tilde{\Sigma}^{m}$ mapped into a simplex $s$ of $S^{m}$ by $f$.) But $\operatorname{deg}\left(f_{1}\right)=i d_{S^{1}}=1(\bmod 2)$ so $f_{k-1}: \tilde{\Sigma}^{k-1} \rightarrow S^{k-1}$ has nonzero degree. Now Proposition 2.4 implies that $\operatorname{deg}(g)=1(\bmod 2)$. Therefore $\left(\tilde{\Sigma}^{k-1}, a\right)$ with equivariant map

$$
\sigma_{k-1}: \tilde{\Sigma}^{k-1} \rightarrow X_{r}
$$

satisfies conditions (i),(ii), and (iii) of Definition 2.3. Now the proof of Proposition 2.6] is complete.

\section{New proper obstructors out of old}

In this Section, we construct a $k$-proper obstructor from a $(k-1)-$ proper obstructor $X$.

Definition 4.1 Let $\left(Y, d_{Y}\right)$ be a proper metric space and $\left(\alpha, d_{\alpha}\right)$ be a metric space isometric to $[0, \infty)$. Let $\phi:[0, \infty) \rightarrow \alpha$ be an isometry and denote $\phi(t)$ by $\alpha_{t}$. Define a metric space $(Y \vee \alpha, d)$, called $Y$ union a ray, as follows:

(i) As a set $Y \vee \alpha$ is the wedge sum. That is, $Y \vee \alpha=Y \cup \alpha$ with $Y \cap \alpha=\left\{\alpha_{0}\right\}$

(ii) The metric $d$ of $Y \vee \alpha$ is defined by

$$
\begin{cases}d(v, w)=d_{Y}(v, w), & \text { if } v, w \in Y \\ d(v, w)=d_{\alpha}(v, w), & \text { if } v, w \in \alpha \\ d(v, w)=d_{Y}\left(v, \alpha_{0}\right)+d_{\alpha}\left(\alpha_{0}, w\right), & \text { if } v \in Y, w \in \alpha\end{cases}
$$


Proposition 4.2 Let $X$ be a $k$-dimensional contractible manifold without boundary and $k>1$. Suppose also that $X$ has a triangulation so that $X$ is a metric simplicial complex and a group $G$ acts on $X$ properly discontinuously, cocompactly, simplicially, and freely by isometries. In particular, $X$ is a $(k-1)-$ proper obstructor. Then $X \vee \alpha$ is a $k$-proper obstructor.

Proof Recall that by Lemma 3.6. we can choose $0<r_{1}<r_{2}<r_{3} \ldots$ and define $\operatorname{pro} \tilde{H}_{k-1}\left(X_{r}\right) \equiv\left\{\tilde{H}_{k-1}\left(X_{r_{i}}\right), i_{*}, \mathbb{N}\right\}$ so that pro $\tilde{H}_{k-1}\left(X_{r}\right)=\mathbb{Z}_{2}$. This means that for any $r>0$ we can choose $R>r$ so that

$$
r^{\prime} \geq R \Rightarrow \mathbb{Z}_{2}=i_{*}\left(H_{k-1}\left(X_{r^{\prime}}\right)\right) \leq H_{k-1}\left(X_{r}\right) .
$$

Now let $r>0$ be given and choose $R>r$ as above. Let $\left(\tilde{\Sigma}^{k-1}, a\right)$ be an essential $\mathbb{Z}_{2}-(k-1)$-cycle with a $\mathbb{Z}_{2}$-equivariant map

$$
f: \tilde{\Sigma}^{k-1} \rightarrow X_{R}
$$

Next consider composition $i \circ f: \tilde{\Sigma}^{k-1} \stackrel{f}{\rightarrow} X_{R} \stackrel{i}{\rightarrow} X_{r}$. If $i \circ f \in Z_{k-1}\left(X_{r}\right)$ is the boundary of a $k$-chain then we can construct an essential $\mathbb{Z}_{2}-k$-cycle with $\mathbb{Z}_{2}-$ equivariant map into $X_{r}$ using the method used in the proof of Proposition 2.6. But this implies $X$ is a $k$-proper obstructor. (Recall that $X^{k}$ is a $(k-1)$ proper obstructor.) So we can assume $i \circ f \in Z_{k-1}\left(X_{r}\right)-B_{k-1}\left(X_{r}\right)$. That is, $0 \neq[i \circ f]=i_{*}[f] \in H_{k-1}\left(X_{r}\right)$. Let $p_{i}: X_{r} \rightarrow X$ denote the projection to the $i$-th factor, $i=1,2$.

We need the following lemma.

Lemma 4.3 Define $j: X-B_{R} \rightarrow X_{R}, x \mapsto\left(\alpha_{0}, x\right)$. Then the composition

$$
i_{*} \circ j_{*}: H_{k-1}\left(X-B_{R}\left(\alpha_{0}\right)\right) \stackrel{j_{*}}{\rightarrow} H_{k-1}\left(X_{R}\right) \stackrel{i_{*}}{\rightarrow} H_{k-1}\left(X_{r}\right)
$$

is nontrivial.

The proof of Lemma 4.3 Consider a map $\lambda: H_{k-1}\left(X_{0}\right) \rightarrow \mathbb{Z}_{2}$ given by

$$
[f] \mapsto L k(f, \Delta)(\bmod 2)
$$

where $L k(f, \Delta)$ denote the linking number of $f$ with the diagonal $\Delta 2$ Now consider the composition:

$$
\zeta: H_{k-1}\left(X-B_{R}\left(\alpha_{0}\right)\right) \stackrel{j_{*}}{\rightarrow} H_{k-1}\left(X_{R}\right) \stackrel{i_{*}}{\rightarrow} H_{k-1}\left(X_{0}\right) \stackrel{\lambda}{\rightarrow} \mathbb{Z}_{2}
$$

\footnotetext{
${ }^{2}$ We can compute $L k(f, \Delta)$ by letting $f$ bound a chain $\tilde{f}$ transverse to $\Delta$ and setting $L k(f, \Delta)=\operatorname{Card}\left(\tilde{f}^{-1}(\Delta)\right)$.
} 
We shall show that $\zeta$ is nontrivial. Choose $\left[f_{1}\right] \in H_{k-1}\left(X-B_{R}\right)$ so that $L k\left(f_{1}, \alpha_{0}\right) \neq 0$ where $\left[\alpha_{0}\right] \in H_{0}(X)$. Then $L k\left(i_{*} j_{*}\left(\left[f_{1}\right]\right), \Delta\right) \neq 0$.(We can choose the same chain transverse to $\Delta$.) Hence $\zeta$ is nontrivial. In particular, $i_{*} \circ j_{*}$ and $j_{*}$ are nontrivial.

Since $j_{*}: H_{k-1}\left(X-B_{R}\right) \rightarrow H_{k-1}\left(X_{R}\right)$ is nontrivial, we can choose $h \in$ $Z_{k-1}\left(X-B_{R}\right)-B_{k-1}\left(X-B_{R}\right)$ with $g \equiv j \circ h \in Z_{k-1}\left(X_{R}\right)-B_{k-1}\left(X_{R}\right)$. That is, $0 \neq[g] \in H_{k-1}\left(X_{R}\right)$. We can consider $g$ as a map $g: \Pi \rightarrow X_{R}$ where $\Pi$ is a $(k-1)$-dimensional simplicial complex satisfying condition (i) of Definition 2.3 such that

- $0 \neq i_{*}[g] \in H_{k-1}\left(X_{r}\right)$

- $i \circ g: \Pi \stackrel{g}{\rightarrow} X_{R} \stackrel{i}{\rightarrow} X_{r}$ with $p_{1}(i \circ g(\Pi))=\left\{\alpha_{0}\right\}=X \cap \alpha(0)$.

Next define $g^{\prime}=s g$, that is,

$$
g^{\prime}: \Pi \stackrel{g}{\rightarrow} X_{R} \stackrel{s}{\rightarrow} X_{R}
$$

Note that $i \circ g^{\prime}$ is a cycle in $X_{R}$ and $p_{2}\left(i \circ g^{\prime}(\Pi)\right)=\alpha_{0}$. Also $[f],[g] \in H_{k-1}\left(X_{R}\right)$ and $i_{*}[f], i_{*}[g] \in H_{k-1}\left(X_{r}\right)$ are nonzero. Observe that $i \circ f$ and $i \circ g$ must be homologous in $X_{r}$ since $\mathbb{Z}_{2}=i_{*}\left(H_{k-1}\left(X_{R}\right)\right) \leq H_{k-1}\left(X_{r}\right)$. We simply write $f$, $g$, and $g^{\prime}$ instead of $i \circ f, i \circ g$, and $i \circ g^{\prime}$. There exists a $k$-chain $G \in C_{k}\left(X_{r}\right)$ such that

$$
\partial G=f+g .
$$

Again consider $G$ as a map $G: \Omega \rightarrow X_{r}$ where $\Omega$ is a simplicial complex so that

$$
\partial \Omega=\tilde{\Sigma}^{k-1} \sqcup \Pi \text {. }
$$

See Figure 4

Next define $G^{\prime}=s G$, that is,

$$
G^{\prime}: \Omega \stackrel{G}{\rightarrow} X_{r} \stackrel{s}{\rightarrow} X_{r}
$$

Note that

$$
\partial G^{\prime}=f+g^{\prime} .
$$

Now take two copies of $\Omega$ and index them as $\Omega_{1}$ and $\Omega_{2}$. Similarly $\Pi_{1} \subset \partial \Omega_{1}$ and $\Pi_{2} \subset \partial \Omega_{2}$. Hence

$$
\partial \Omega_{i}=\tilde{\Sigma}^{k-1} \cup \Pi_{i}, i=1,2 .
$$




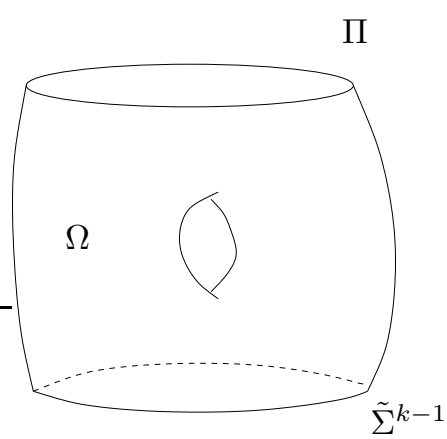

Figure 4: $\Omega$

Denote $i d(x)=x^{\prime}$ for $x \in \Omega_{1}-\tilde{\Sigma}^{k}$ where $i d: \Omega_{1} \rightarrow \Omega_{2}$. Construct a $k-$ dimensional simplicial complex $\tilde{\Omega}$ by attaching $\Omega_{1}$ and $\Omega_{2}$ along $\tilde{\Sigma}^{k-1}$ by $a: \tilde{\Sigma}^{k-1} \rightarrow \tilde{\Sigma}^{k-1}$. That is,

$$
\tilde{\Omega}=\left(\Omega_{1} \cup \Omega_{2}\right) / x \sim a x, x \in \tilde{\Sigma}^{k-1} .
$$

See Figure 5

We can define an involution $\bar{a}$ on $\tilde{\Omega}$ by

$$
\begin{cases}\bar{a}(x)=a(x), & x \in \tilde{\Sigma}^{k-1}, \\ \bar{a}(x)=x^{\prime}, & x \in \Omega_{1}-\tilde{\Sigma}^{k-1}, \\ \bar{a}\left(x^{\prime}\right)=x, & x^{\prime} \in \Omega_{2}-\tilde{\Sigma}^{k-1} .\end{cases}
$$

Also we can define a $\mathbb{Z}_{2}$-equivariant map $\Phi: \tilde{\Omega} \rightarrow X_{r}$ by:

$$
\left\{\begin{array}{l}
\left.\Phi\right|_{\Omega_{1}}=G \\
\left.\Phi\right|_{\Omega_{2}}=G^{\prime}
\end{array}\right.
$$

We define

$$
\tilde{\Sigma}^{k}=\left(\Pi_{1} \times[0,1] /\left(\Pi_{1}, 1\right) \sim *\right) \cup_{\Pi_{1}} \tilde{\Omega} \cup_{\Pi_{2}}\left(\Pi_{2} \times[0,-1] /\left(\Pi_{2},-1\right) \sim *\right) .
$$

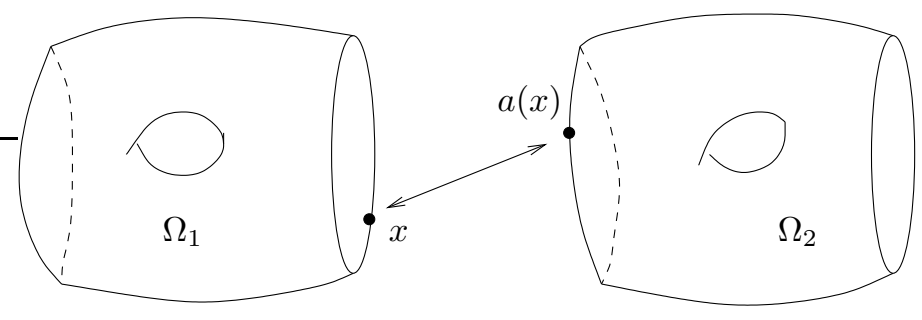

Figure 5: Constructing $\tilde{\Omega}$ 
See Figure 6. Now extend $\bar{a}$ over $\tilde{\Sigma}^{k}$, and denote $\tilde{\Sigma}^{k} / x \sim a(x)$ by $\Sigma^{k}$.

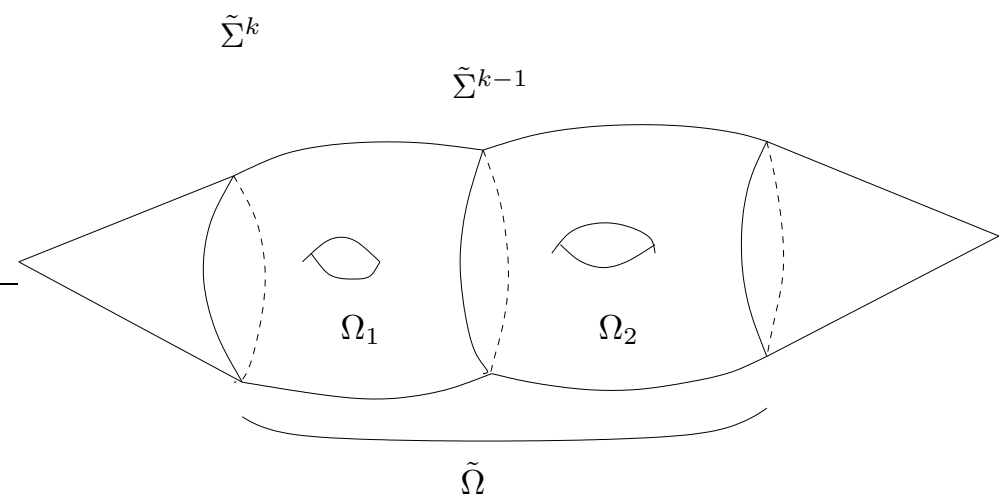

Figure 6: Constructing $\tilde{\Sigma}^{k}$

Suppose that $\Sigma^{k}$ classifies into $\mathbb{R} P^{m}$ where $m<k$. Let

$$
h: \Sigma^{k} \rightarrow \mathbb{R} P^{m}
$$

be the classifying map and

$$
\tilde{h}: \tilde{\Sigma}^{k} \rightarrow S^{m}
$$

be the equivariant map covering $h$. Observe that

$$
\left.\operatorname{deg} \tilde{h}\right|_{\tilde{\Sigma}^{k-1}}=\operatorname{deg} \tilde{h}=0(\bmod 2)
$$

This is a contradiction since there already exists a $\mathbb{Z}_{2}$-equivariant map

$$
\varphi: \tilde{\Sigma}^{k-1} \rightarrow S^{k-1}
$$

of odd degree. Hence $\left(\tilde{\Sigma}^{k}, \bar{a}\right)$ is an essential $\mathbb{Z}_{2}-k$-cycle.

Finally, we need to define a $\mathbb{Z}_{2}$-equivariant map:

$$
F: \tilde{\Sigma}^{k} \rightarrow(X \vee \alpha)_{r}
$$

Recall that $p_{1} g(\Pi)=\alpha_{0}$ and let

$$
c: p_{2} g\left(\Pi_{1}\right) \times I \rightarrow X
$$

be a contraction to $\alpha_{0}$. Similarly $p_{2} g^{\prime}(\Pi)=\alpha_{0}$ and let

$$
c^{\prime}: p_{1} g^{\prime}\left(\Pi_{1}\right) \times I \rightarrow X
$$

be a contraction to $\alpha_{0}$. Define a $\mathbb{Z}_{2}$-equivariant map

$$
F: \tilde{\Sigma}^{k} \rightarrow(X \vee \alpha)_{r}
$$


as follows: Recall that $\phi(t)=\alpha_{t}$ in Definition 4.1 so $d\left(\alpha_{0}, \alpha_{s}\right)=s$ and $d\left(\alpha_{s}, x\right) \geq s$ for any $x \in X$.

$$
\begin{cases}\left.F\right|_{\tilde{\Omega}}=\Phi & \\ F(x, t)=\left(\alpha_{2 r t}, p_{2} g(x)\right), & x \in \Pi_{1}, t \in\left[0, \frac{1}{2}\right] \\ F(x, t)=\left(\alpha_{r}, c_{(2 t-1)}\left(p_{2} g(x)\right)\right), & x \in \Pi_{1}, t \in\left[\frac{1}{2}, 1\right] \\ F(x, t)=\left(p_{1} g^{\prime}(x), \alpha_{-2 r t}\right), & x \in \Pi_{2}, t \in\left[0,-\frac{1}{2}\right] \\ F(x, t)=\left(c_{(-2 t-1)}\left(p_{1} g^{\prime}(x)\right), \alpha_{r}\right), & x \in \Pi_{2}, t \in\left[-\frac{1}{2},-1\right]\end{cases}
$$

The proof of Proposition 4.2 is now complete.

If $Y$ and $Z$ are metric spaces we use the sup metric on $Y \times Z$ where

$$
d_{\text {sup }}\left(\left(y_{1}, z_{1}\right),\left(y_{2}, z_{2}\right)\right) \equiv \max \left\{d_{Y}\left(y_{1}, y_{2}\right), d_{Z}\left(z_{1}, z_{2}\right)\right\} .
$$

Proposition 4.4 Suppose $X_{1}, X_{2}$ are $m_{1}, m_{2}$-proper obstructors, respectively. Then $X_{1} \times X_{2}$ is an $\left(m_{1}+m_{2}+1\right)$-proper obstructor.

Proof Let $r>0$ be given and let

$$
\left\{\begin{array}{l}
f_{1}: \tilde{\Sigma}_{1}^{m_{1}} \rightarrow\left(X_{1}\right)_{r} \\
f_{2}: \tilde{\Sigma}_{2}^{m_{2}} \rightarrow\left(X_{2}\right)_{r}
\end{array}\right.
$$

be $\mathbb{Z}_{2}$-equivariant maps for essential $\mathbb{Z}_{2}$-cycles. Note that

$$
\left(X_{1} \times X_{2}\right)_{r}=\left(\left(X_{1}\right)_{r} \times\left(X_{2}\right)^{2}\right) \cup_{\left(X_{1}\right)_{r} \times\left(X_{2}\right)_{r}}\left(\left(X_{1}\right)^{2} \times\left(X_{2}\right)_{r}\right) .
$$

Let $a_{1}$ be the involution on $\left(X_{1}\right)_{r}$ and $a_{2}$ be the involution on $\left(X_{2}\right)_{r}$. Recall that the join $\tilde{\Sigma}_{1}^{m_{1}} * \tilde{\Sigma}_{2}^{m_{2}}$ is obtained from $\tilde{\Sigma}_{1}^{m_{1}} \times \tilde{\Sigma}_{2}^{m_{2}} \times[-1,1]$ by identifying $\tilde{\Sigma}_{1}^{m_{1}} \times\{y\} \times\{1\}$ to a point for every $y \in \tilde{\Sigma}_{2}^{m_{2}}$ and identifying $\{x\} \times \tilde{\Sigma}_{2}^{m_{2}} \times\{-1\}$ to a point for every $x \in \tilde{\Sigma}_{1}^{m_{1}}$. Define an involution $a$ on $\tilde{\Sigma}_{1}^{m_{1}} * \tilde{\Sigma}_{2}^{m_{2}}$ by

$$
a(v, w, t)=\left(a_{1}(v), a_{2}(w), t\right) .
$$

Let

$$
\left\{\begin{array}{l}
g_{1}: \tilde{\Sigma}_{1}^{m_{1}} \rightarrow S^{m_{1}} \\
g_{2}: \tilde{\Sigma}_{2}^{m_{2}} \rightarrow S^{m_{2}}
\end{array}\right.
$$

be equivariant maps of odd degree. Then:

$$
\begin{aligned}
g_{1} * g_{2}: \tilde{\Sigma}_{1}^{m_{1}} * \tilde{\Sigma}_{2}^{m_{2}} & \rightarrow S^{m_{1}} * S^{m_{2}}=S^{m_{1}+m_{2}+1} \\
(v, w, t) & \mapsto\left(g_{1}(v), g_{2}(w), t\right)
\end{aligned}
$$

is also an equivariant map of an odd degree. Hence $\left(\tilde{\Sigma}_{1}^{m_{1}} * \tilde{\Sigma}_{2}^{m_{2}}, a\right)$ is an essential $\mathbb{Z}_{2}-\left(m_{1}+m_{2}+1\right)$-cycle. 
Now let

$$
c: f_{1}\left(\tilde{\Sigma}_{1}^{m_{1}}\right) \times[-1,1] \rightarrow X_{1}^{2}
$$

be a $\mathbb{Z}_{2}$-equivariant contraction to a point such that $c_{t}=i d$ for $t \in[-1,0]$. Similarly let

$$
d: f_{2}\left(\tilde{\Sigma}_{2}^{m_{2}}\right) \times[-1,1] \rightarrow X_{2}^{2}
$$

be a $\mathbb{Z}_{2}$-equivariant contraction to a point such that $d_{t}=i d$ for $t \in[0,1]$.

Finally define

$$
f: \tilde{\Sigma}_{1}^{m_{1}} * \tilde{\Sigma}_{2}^{m_{2}} \rightarrow\left(X_{1} \times X_{2}\right)_{r} \text { by } f(v, w, t)=\left(c_{t}\left(f_{1}(v)\right), d_{t}\left(f_{2}(w)\right)\right) .
$$

We note that $f$ is $\mathbb{Z}_{2}$-equivariant.

\section{Proper obstructor dimension}

We review one more notion from [2].

Definition 5.1 The uniformly proper dimension, updim $(G)$, of a discrete group $G$ is the smallest integer $m$ such that there is a contractible $m$-manifold $W$ equipped with a proper metric $d_{W}$, and there is a $g: \Gamma \rightarrow W$ with the following properties:

- $g$ is Lipschitz and uniformly proper.

- There is a function $\rho:(0, \infty) \rightarrow(0, \infty)$ such that any ball of radius $r$ centered at a point of the image of $h$ is contractible in the ball of radius $\rho(r)$ centered at the same point.

If no such $n$ exists, we define $\operatorname{updim}(G)=\infty$.

It was proved in [2] that

$$
\operatorname{updim}(G) \leq \operatorname{actdim}(G) .
$$

Now we generalize the obstructor dimension of a group.

Definition 5.2 The proper obstructor dimension of $G$, pobdim $(G)$, is defined to be 0 for finite groups, 1 for 2 -ended groups, and otherwise $m+1$ where $m$ is the largest integer such that for some $m$-proper obstructor $Y$, there exists a uniformly proper map

$$
\phi: Y \rightarrow T_{G}
$$

where $T_{G}$ is a proper metric space with a quasi-isometry $q: T_{G} \rightarrow G$. 
Lemma 5.3 Let $Y$ be an $m$-proper obstructor. If there is a uniformly proper map $\beta: Y \rightarrow W^{d}$ where $W$ is a contractible $d$-manifold then $d>m$.

Proof Assume $d \leq m(d-1 \leq m-1)$. Observe that if $\beta$ is uniformly proper then $\beta$ induces an equivariant map $\bar{\beta}: Y_{r} \rightarrow W_{0}$ for some large $r>0$. Now let $f: \tilde{\Sigma}^{m} \rightarrow Y_{r}$ be an essential $\mathbb{Z}_{2}-m-$ cycle where $f$ is equivariant. Let $h: W_{0} \rightarrow$ $S^{d-1}$ be an equivariant homotopy equivalence. We have an equivariant map

$$
g=i h \bar{\beta} f: \tilde{\Sigma}^{m} \stackrel{f}{\rightarrow} Y_{r} \stackrel{\bar{\beta}}{\rightarrow} W_{0} \stackrel{h}{\rightarrow} S^{d-1} \stackrel{i}{\rightarrow} S^{m-1} \stackrel{i}{\rightarrow} S^{m}
$$

where $i: S^{d-1} \rightarrow S^{m-1} \rightarrow S^{m}$ is the inclusion. Note that $g$ is equivariant but $\operatorname{deg}(g)=0(\bmod 2)$. This is a contradiction by Proposition 2.4 .

Suppose that $G$ is finite so that $\operatorname{pobdim}(G)=0$ by definition.

Clearly, $\operatorname{actdim}(G)=0$ if $G$ is finite. Hence $\operatorname{pobdim}(G)=\operatorname{actdim}(G)=0$ in this case. Next suppose that $G$ has two ends so that $\operatorname{pobdim}(G)=1$. Note that there exists $\mathbb{Z} \cong H \leq G$ with $|G: H|<\infty$. And this implies that

$$
\operatorname{actdim}(G)=\operatorname{actdim}(H)=\operatorname{actdim}(\mathbb{Z})=1 .
$$

Therefore, $\operatorname{pobdim}(G)=\operatorname{actdim}(G)=1$ when $G$ has two ends. Now we prove the main theorem for the general case.

Main Theorem $\operatorname{pobdim}(G) \leq \operatorname{updim}(G) \leq \operatorname{actdim}(G)$

Proof We only need to show the first inequality. Let $\operatorname{pobdim}(G)=m+1$ for some $m>0$. That is, there exists an $m$-proper obstructor $Y$, a proper metric space $T_{G}$, a uniformly proper map $\psi: Y \rightarrow T_{G}$, and a quasi-isometry $q: T_{G} \rightarrow G$. Let updim $(G) \equiv d$ such that there exists a uniformly proper map $\phi: G \rightarrow W^{d}$ where $W$ is a contractible $d$-manifold. But the composition

$$
\phi \circ q \circ \psi: Y \rightarrow T_{G} \rightarrow G \rightarrow W^{d}
$$

is uniformly proper. Therefore

$$
m+1=\operatorname{pobdim}(G) \leq \operatorname{updim}(G)
$$

by the previous lemma.

Before we consider some applications, we make the following observation about compact aspherical manifolds with incompressible boundary. 
Lemma 5.4 Assume that $W$ is a compact aspherical $m$-manifold with all boundary components incompressible. Let $\pi: \tilde{W} \rightarrow W$ denote the universal cover of $W$. Suppose that there is a component of $\partial W$, call it $N$, so that $\left|\pi_{1}(W): \pi_{1}(N)\right|>2$. Then $\left|\pi_{1}(W): \pi_{1}(N)\right|$ is infinite.

Proof Observe that $N$ is aspherical also. First, we show that if

$$
1<\left|\pi_{1}(W): \pi_{1}(N)\right|<\infty
$$

then $\tilde{M} \equiv \tilde{W} / \pi_{1}(N)$ has two boundary components and $W$ has one boundary component. We claim that $\tilde{M}$ has a boundary component homeomorphic to $N$ which is still denoted by $N$. To see this consider the long exact sequence:

$$
\cdots \rightarrow H_{1}(\partial \tilde{M}) \stackrel{i_{*}}{\rightarrow} H_{1}(\tilde{M}) \rightarrow H_{1}(\tilde{M}, \partial \tilde{M}) \rightarrow \tilde{H}_{0}(\partial \tilde{M}) \rightarrow \tilde{H}_{0}(\tilde{M})=0
$$

Since $\pi_{1}(N)=\pi_{1}(\tilde{M}), i_{*}: H_{1}(\partial \tilde{M}) \rightarrow H_{1}(\tilde{M})$ is surjective. So we have:

$$
0 \rightarrow H_{1}(\tilde{M}, \partial \tilde{M}) \rightarrow \tilde{H}_{0}(\partial \tilde{M}) \rightarrow 0
$$

Since $\left|\pi_{1}(W): \pi_{1}(N)\right|$ is finite $\tilde{M}$ is compact. Now $H_{1}(\tilde{M}, \partial \tilde{M}) \cong H^{m-1}(\tilde{M})$ by duality. But $H^{m-1}(\tilde{M}) \cong H^{m-1}(N)$ and $H^{m-1}(N) \cong \mathbb{Z}_{2}$ since $N$ is a closed $(m-1)$-manifold. That is, $\tilde{H}_{0}(\partial \tilde{M}) \cong \mathbb{Z}_{2}$ so $\tilde{M}$ has two boundary components. Next let $N$ and $N^{\prime}$ denote two boundary components of $\partial \tilde{M}$ both of which are mapped to $N \subset W$ by $p: \tilde{M} \rightarrow W$. Hence $\partial W$ has one component.

Now assume that $m \equiv\left|\pi_{1}(W): \pi_{1}(N)\right|>2$. Suppose $m$ is finite. Note that $\left.p\right|_{N}: N(\subset \tilde{M}) \rightarrow N(\subset W)$ has index 1 , and $\left.p\right|_{N^{\prime}}: N^{\prime}(\subset \tilde{M}) \rightarrow N(\subset W)$ has index $m-1$. This means that $\left|\pi_{1}(\tilde{M}): \pi_{1}\left(N^{\prime}\right)\right|=m-1$ since $\pi_{1}(\tilde{M})=\pi_{1}(N)$. There are two alternative arguments:

- If $m>2$ then $\tilde{M}$ is an aspherical manifold with two boundary components $N$ and $N^{\prime}$ with $\left|\pi_{1}(\tilde{M}): \pi_{1}\left(N^{\prime}\right)\right|=m-1>1$. Consider $\tilde{W} / \pi_{1}\left(N^{\prime}\right)$. The same argument applied to $\tilde{W} / \pi_{1}\left(N^{\prime}\right)$ shows that $\tilde{M}$ has one boundary component, which is a contradiction. Therefore $\left|\pi_{1}(W): \pi_{1}(N)\right|$ is infinite.

- Suppose $m>2$. Choose a point $x \in N \subset \partial W$ and let $\tilde{x} \in N \subset \partial \tilde{M}$ so that $p(\tilde{x})=x$. Next choose two loops $\alpha$ and $\beta$ in $W$ based at $x$ so that $\left\{\pi_{1}(N),[\alpha] \pi_{1}(N),[\beta] \pi_{1}(N)\right\}$ are distinct cosets. (We are assuming $\left|\pi_{1}(W): \pi_{1}(N)\right|>2$.) Let $\tilde{\alpha}$ and $\tilde{\beta}$ be the liftings of $\alpha$ and $\beta$ respectively so that $\tilde{\alpha}(0)=\tilde{x}=\tilde{\beta}(0)$. Note that $\tilde{y}_{1} \equiv \tilde{\alpha}(1), \tilde{y}_{2} \equiv \tilde{\beta}(1) \in N^{\prime}$ and $\tilde{y}_{1} \neq \tilde{y}_{2}$ since $[\alpha] \pi_{1}(N) \neq[\beta] \pi_{1}(N)$. Now consider a path $\tilde{\gamma}$ in $N^{\prime}$ from $\tilde{y}_{1}$ to $\tilde{y}_{2}$. Observe that $p \tilde{\gamma} \equiv \gamma$ is a loop based at $x$, and $[\gamma] \in p_{*}\left(\pi_{1}\left(N^{\prime}\right)\right) \leq \pi_{1}(N)$. But $[\alpha][\gamma][\beta]^{-1}=1$ and this implies that $[\alpha]^{-1}[\beta] \in \pi_{1}(N)$ contary to $[\alpha] \pi_{1}(N) \neq[\beta] \pi_{1}(N)$. 
Corollary 5.5 (Application) Suppose that $W$ is a compact aspherical $m$ manifold with incompressible boundary. Also assume that there is a component of $\partial W$, call it $N$, so that $\left|\pi_{1}(W): \pi_{1}(N)\right|>2$.

Then $\operatorname{actdim}\left(\pi_{1}(W)\right)=m$.

Proof Let $p: \tilde{W} \rightarrow W$ be the universal cover of $W$. It is obvious that $\operatorname{actdim}\left(\pi_{1}(W)\right) \leq m$ as $\pi_{1}(W)$ acts cocompactly and properly discontinuously on $\tilde{W}$. Denote $G \equiv \pi_{1}(W)$ and $H \equiv \pi_{1}(N)$. Let $\tilde{N}$ be a component of $p^{-1}(N)$. Therefore $\tilde{N}$ is the contractible universal cover of $N^{(m-1)}$. Note that $\tilde{N}$ is an $(m-2)$-proper obstructor by Proposition 2.6. Now $\tilde{W} / H$ has a boundary component homeomorphic to $N$. Call this component $N$ also. $|G: H|$ is infinite by the previous lemma, and this implies that $\tilde{W} / H$ is not compact. In particular, there exists a map $\alpha^{\prime}:[0, \infty) \rightarrow \tilde{W} / H$ with the following property: For each $D>0$ there exists $T \in[0, \infty)$ such that for any $x \in N, d\left(\alpha^{\prime}(t), x\right)>D$ for $t>T$, and $\alpha^{\prime}(0) \in N$. Let $\tilde{\alpha}:[0, \infty) \rightarrow \tilde{W}$ be a lifting of $\alpha^{\prime}$ such that $\tilde{\alpha}(0) \in \tilde{N}$. Now we define a uniformly proper map:

$$
\begin{gathered}
\phi: \tilde{N} \vee \alpha \rightarrow \tilde{W} \\
\left\{\begin{array}{l}
\left.\phi\right|_{\tilde{N}}=\text { inclusion } \\
\phi\left(\alpha_{t}\right)=\tilde{\alpha}(t)
\end{array}\right.
\end{gathered}
$$

Observe that $\phi$ is a uniformly proper map. Since $\tilde{N} \vee \alpha$ is an $(m-1)$-proper obstructor and $\tilde{W}$ is quasi-isometric to $G, \operatorname{pobdim}(G) \geq m$. But

$$
\operatorname{pobdim}(G) \leq \operatorname{updim}(G) \leq \operatorname{actdim}(G) \leq m .
$$

The last inequality follows from the fact that $G$ acts on $\tilde{W}$ properly discontinuously. Therefore $\operatorname{pobdim}(G)=m$.

The following corollary answers Question 2 found in [2].

Corollary 5.6 (Application) Suppose that $W_{i}$ is a compact aspherical $m_{i}-$ manifold with incompressible boundary for $i=1, \ldots, d$. Also assume that for each $i, 1 \leq i \leq d$, there is a component of $\partial W_{i}$, call it $N_{i}$, so that $\left|\pi_{1}\left(W_{i}\right): \pi_{1}\left(N_{i}\right)\right|>2$. Let $G \equiv \pi_{1}\left(W_{1}\right) \times \ldots \times \pi_{1}\left(W_{d}\right)$. Then:

$$
\operatorname{actdim}(G)=m_{1}+\ldots+m_{d}
$$

Proof It is easy to see that

$$
\operatorname{actdim}(G) \leq m_{1}+\ldots+m_{d}
$$


as $G$ acts cocompactly and properly discontinuously on $\tilde{W}_{1} \times \cdots \times \tilde{W}_{d}$. Denote $\pi_{1}\left(W_{i}\right) \equiv G_{i}$ and $\pi_{1}\left(N_{i}\right) \equiv H_{i}$. Let

$$
p: \tilde{W}_{i} \rightarrow W_{i}
$$

be the contractible universal cover and let $\tilde{N}_{i}$ be a component of $p^{-1}\left(N_{i}\right)$. Since $N_{i}$ is incompressible, $\tilde{N}_{i}$ is the contractible universal cover of $N_{i}^{\left(m_{i}-1\right)}$.

By the previous Corollary, there are uniformly proper maps:

$$
\begin{aligned}
& \phi_{1}: \tilde{N}_{1} \vee \alpha \rightarrow \tilde{W}_{1} \\
& \phi_{2}: \tilde{N}_{2} \vee \beta \rightarrow \tilde{W}_{2}
\end{aligned}
$$

So there exists a uniformly proper map:

$$
\phi_{1} \times \phi_{2}:\left(\tilde{N}_{1} \vee \alpha\right) \times\left(\tilde{N}_{2} \vee \beta\right) \rightarrow \tilde{W}_{1} \times \tilde{W}_{2}
$$

Recall that $\left(\tilde{N}_{1} \vee \alpha\right) \times\left(\tilde{N}_{2} \vee \beta\right)$ is an $\left(m_{1}+m_{2}-1\right)$-proper obstructor by Proposition 4.4 Since $\tilde{W}_{1} \times \tilde{W}_{2}$ is quasi-isometric to $G_{1} \times G_{2}$ :

$$
\operatorname{pobdim}\left(G_{1} \times G_{2}\right) \geq m_{1}+m_{2}
$$

But $G_{1} \times G_{2}$ acts on $\tilde{W}_{1} \times \tilde{W}_{2}$ properly discontinuously, and this implies that:

$$
\operatorname{pobdim}\left(G_{1} \times G_{2}\right) \leq \operatorname{actdim}\left(G_{1} \times G_{2}\right) \leq m_{1}+m_{2}
$$

Therefore, $\operatorname{pobdim}\left(G_{1} \times G_{2}\right)=m_{1}+m_{2}$.

Continue inductively and we conclude that:

$$
\operatorname{pobdim}(G)=\operatorname{pobdim}\left(G_{1} \times \cdots \times G_{d}\right)=m_{1}+\ldots+m_{d}
$$

Finally we see that

$$
\operatorname{pobdim}(G) \leq \operatorname{updim}(G) \leq \operatorname{actdim}(G) \Rightarrow \operatorname{actdim}(G)=m_{1}+\ldots+m_{d}
$$

Acknowledgements The author thanks Professor M. Bestvina for numerous helpful discussions.

\section{A Pro-Category of Abelian Groups}

With every category $\mathcal{K}$ we can associate a new category pro $(\mathcal{K})$. We briefly review the definitions, see [1] or [6] for details. Recall that a partially ordered set $(\Lambda, \leq)$ is directed if, for $i, j \in \Lambda$, there exists $k \in \Lambda$ so that $k \geq i, j$. 
Definition A.1 (Inverse system) Let $(\Lambda, \leq)$ be a directed set. The system $\mathbf{A}=\left\{A_{\lambda}, p_{\lambda}^{\lambda^{\prime}}, \Lambda\right\}$ is called an inverse system over $(\Lambda, \leq)$ in the category $\mathcal{K}$, if the following conditions are true.

(i) $A_{\lambda} \in O b_{\mathcal{K}}$ for every $\lambda \in \Lambda$

(ii) $p_{\lambda}^{\lambda^{\prime}} \in \operatorname{Mor}_{\mathcal{K}}\left(A_{\lambda^{\prime}}, A_{\lambda}\right)$ for $\lambda^{\prime} \geq \lambda$

(iii) $\lambda \leq \lambda^{\prime} \leq \lambda^{\prime \prime} \Rightarrow p_{\lambda}^{\lambda^{\prime}} p_{\lambda^{\prime}}^{\lambda^{\prime \prime}}=p_{\lambda}^{\lambda^{\prime \prime}}$

Definition A.2 (A map of systems) Given two inverse systems in $\mathcal{K}$,

$$
\mathbf{A}=\left\{A_{\lambda}, p_{\lambda}^{\lambda^{\prime}}, \Lambda\right\}, \text { and } \mathbf{B}=\left\{B_{\mu}, q_{\mu}^{\mu^{\prime}}, M\right\}
$$

the system

$$
\bar{f}=\left(f, f_{\lambda}\right): \mathbf{A} \rightarrow \mathbf{B}
$$

is called a map of systems if the following conditions are true.

(i) $f: M \rightarrow \Lambda$ is an increasing function

(ii) $f(M)$ is cofinal with $\Lambda$

(iii) $f_{\mu} \in \operatorname{Mor}_{\mathcal{K}}\left(A_{f(\mu)}, B_{\mu}\right)$

(iv) For $\mu^{\prime} \geq \mu$ there exists $\lambda \geq f(\mu), f\left(\mu^{\prime}\right)$ so that:

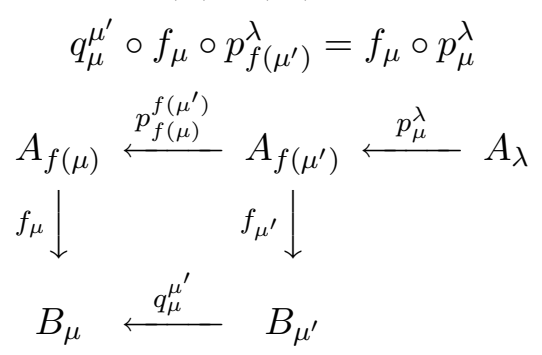

$\bar{f}$ is called a special map of systems if $\Lambda=M, f=i d$, and $f_{\lambda} p_{\lambda}^{\lambda^{\prime}}=q_{\lambda}^{\lambda^{\prime}} f_{\lambda^{\prime}}$. Two maps of systems $\bar{f}, \bar{g}: \mathbf{A} \rightarrow \mathbf{B}$ are considered equivalent, $\bar{f} \simeq \bar{g}$, if for every $\mu \in M$ there is a $\lambda \in \Lambda, \lambda \geq f(\mu), g(\mu)$, such that $f_{\mu} p_{f(\mu)}^{\lambda}=g_{\mu} p_{g(\mu)}^{\lambda}$. This is an equivalence relation.

Definition A.3 (Pro-category) pro $(\mathcal{K})$ is a category whose objects are inverse systems in $\mathcal{K}$ and morphisms are equivalence classes of maps of systems. The class containing $\bar{f}$ will be denoted by $\mathbf{f}$.

Our main interest is the following pro-category.

Example A.4 Pro-category of abelian groups Let $\mathcal{A}$ be the category of abelian groups and homomorphisms. Then corresponding $\operatorname{pro}(\mathcal{A})$ is called the category of pro-abelian groups. 
Example A.5 Homology pro-groups Suppose $\left\{\left(X, X_{0}\right)_{i}, p_{i}^{i^{\prime}}, \mathbb{N}\right\}$ is an object in the pro-homotopy category of pairs of spaces having the homotopy type of a simplicial pair. Then $\left\{H_{j}\left(\left(X, X_{0}\right)_{i}\right),\left(p_{i}^{i^{\prime}}\right)_{*}, \mathbb{N}\right\}$ is an object of $\operatorname{pro}(\mathcal{A})$. Denote $\left\{H_{j}\left(\left(X, X_{0}\right)_{i}\right),\left(p_{i}^{i^{\prime}}\right)_{*}, \mathbb{N}\right\}$ by $\operatorname{proH}_{j}\left(X, X_{0}\right)$.

We list useful properties of $\operatorname{pro}(\mathcal{A})$ :

(1) A system $\mathbf{0}$ consisting of a single trivial group is a zero object in $\operatorname{pro}(\mathcal{A})$.

(2) A pro-abelian group $\left\{G_{i}, p_{i}^{i^{\prime}}, \mathbb{N}\right\} \cong \mathbf{0}$ iff every $i$ admits a $i^{\prime} \geq i$ such that $p_{i}^{i^{\prime}}=0$.

(3) Let $\mathbf{A}$ denote a constant pro-abelian group $\left\{A, i d_{A}, \mathbb{N}\right\}$. If a pro-abelian group $\left\{G_{i}, p_{i}^{i^{\prime}}, \mathbb{N}\right\} \cong \mathbf{A}$ then

$$
\lim _{\leftarrow} G_{i}=A .
$$

See [4, Lemma 4.1].

\section{References}

[1] M. Artin, B. Mazur Etale Homotopy, Springer-Verlag, (1969).

[2] M. Bestvina, M. Kapovich, B. Kleiner van Kampen's embedding obstructions for discrete groups, Invent. Math. (2) 150, 219-235 (2002).

[3] K. Brown, Cohomology of Groups, Springer-Verlag, (1994).

[4] D. Edwards, R Geoghegan The stability problem in shape, and a Whitehead theorem in pro-homotopy, Trans. of AMS. 214, 261-277 (1975).

[5] M. Kapovich, B. Kleiner, Coarse Alexander duality and duality groups, preprint, (2001).

[6] S. Mardešić, On the Whitehead theorem in shape theory I, Fund. Math. 91, 51-64 (1976).

[7] A. Shapiro, Obstructions to the embedding of a complex in a Euclidean space I. The first obstruction, Ann. of Math. (2) 66, 256-269 (1957).

[8] E. R. van Kampen, Komplexe in euklidischen Raumen, Abh. Math. Sem. Univ. Hamburg 9, 72-78 and 152-153 (1933).

110 8th Street RPI, Troy, NY 12180, USA

Email: yoons@rpi.edu

Received: 28 March 2003 